\title{
Maintaining memory of silencing at imprinted differentially methylated regions
}

\author{
Hsiao P. J. Voon ${ }^{1} \cdot$ Richard J. Gibbons $\left.^{2,3}\right]_{(}$
}

Received: 13 December 2015/Accepted: 4 February 2016/Published online: 16 February 2016

(C) The Author(s) 2016. This article is published with open access at Springerlink.com

\begin{abstract}
Imprinted genes are an exceptional cluster of genes which are expressed in a parent-of-origin dependent fashion. This allele-specific expression is dependent on differential DNA methylation which is established in the parental germlines in a sex-specific manner. The DNA methylation imprint is accompanied by heterochromatin modifications which must be continuously maintained through development. This review summarises the factors which are important for protecting the epigenetic modifications at imprinted differentially methylated regions (DMRs), including PGC7, ZFP57 and the ATRX/Daxx/ H3.3 complex. We discuss how these factors maintain heterochromatin silencing, not only at imprinted DMRs, but also other heterochromatic regions in the genome.
\end{abstract}

Keywords Genomic imprinting - Heterochromatin . PGC7 $\cdot$ Zfp57 $\cdot$ ATRX $\cdot$ Daxx $\cdot$ H3.3

Richard J. Gibbons

richard.gibbons@imm.ox.ac.uk

Hsiao P. J. Voon

joanna.voon@monash.edu

1 Department of Biochemistry and Molecular Biology, Monash University, Clayton, VIC 3800, Australia

2 University of Oxford, Oxford, UK

3 MRC Molecular Haematology Unit, Weatherall Institute of Molecular Medicine, John Radcliffe Hospital, Headington, Oxford OX3 9DS, UK

\section{Introduction}

Imprinted genes are a specialised group of genes in mammalian genomes which are monoallelically expressed in a parent-of-origin dependent manner. Approximately 100-150 imprinted genes have been identified in both the mouse and the human genome to date [1]. The majority of these genes are arranged in chromosomal clusters and imprinted monoallelic expression of multiple genes in a cluster can be controlled by a single germline differentially methylated region (gDMR) within the cluster [2-5]. The differential methylation at gDMRs is established in parental germlines and maintained through development to facilitate imprinted gene expression. Of the 20 gDMRs identified to date, only three gDMRs acquire DNA methylation in the paternal germline while the remainder are methylated on the maternally inherited allele [6]. The maternal and paternal gDMRs are associated with distinct genomic locations; the three paternally methylated gDMRs are located at intergenic sites while maternally methylated gDMRs are $\mathrm{CpG}$ islands (CGIs) which are intragenic and embedded within transcriptional units [6] (Fig. 1). The different positions of maternal vs paternal gDMRs possibly arises from sex-specific differences in germ cell development including differences in gene expression, timing of methylation and rounds of cell division.

In addition to differential DNA methylation, gDMRs are also associated with differential histone modifications in accordance with the methylation status [7-10]. The unmethylated allele is enriched for modifications associated with active chromatin such as H3K4me3 [8, 10-12], which also protects against de novo DNA methylation. Conversely, the methylated allele is enriched for modifications associated with constitutive heterochromatin such as $\mathrm{H} 3 \mathrm{~K} 9 \mathrm{me} 2 / 3[8,10,12]$. These modified histones are 

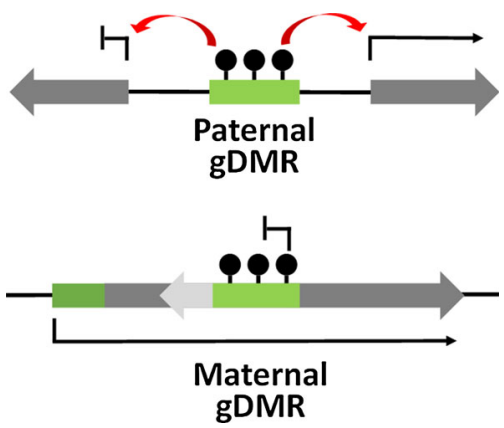

Fig. 1 Paternal and maternal methylated gDMRs are associated with distinct genomic locations. Paternally methylated gDMRs are located at intergenic regions and methylation at gDMRs specifies allelespecific expression of genes at flanking genes. Maternally methylated gDMRs are located at intragenic sites and methylation can directly repress transcription of an antisense transcript as well as influence the expression of flanking genes within the cluster

present in both sperm and oocytes and contribute to the maintenance of imprinting through development.

To correctly specify imprinted gene expression in the developing organisms, DNA methylation at gDMRs must survive a wave of demethylation which occurs during very early development [13] (Fig. 2). Mammalian genomes undergo two cyclical waves of demethylation during development. The first wave of demethylation occurs postfertilisation in the pre-implantation embryo. The paternal genome is subjected to active demethylation during this period while the maternal genome undergoes passive demethylation [14]. The imprinted gDMRs and certain repeats are protected from this indiscriminate demethylation, and DNA methylation across the genome is subsequently restored during development. The second wave of demethylation occurs during germline specification in primordial germ cells when the entire genome (including gDMRs) is demethylated with the exception of IAP retrotransposon repeats in the mouse $[15,16]$. This resets the genome and methylation at gDMRs is then re-established in a sex-specific manner in the maturing germ cells [17, 18]. It is worth nothing that differential methylation at this stage is not limited to gDMRs but is instead prevalent across the genome [17, 18]. Much of the differential methylation acquired in the germline is subsequently lost during the wave of preimplantation demethylation, with the exception of imprinted DMRs and certain repeats (e.g. IAPs) [17, 18]. Protection of DNA methylation at gDMRs is critical during the pre-implantation period and two proteins, PGC7 and Zfp57, are known to be important for this process.

\section{PGC7 protects imprinted methylated DMRs from passive demethylation}

PGC7 (also known as Stella or Dppa3) was first identified as a protein which is highly expressed in primordial germ cells (PGCs) [19]. Expression of PGC7 is maintained through to oocyte maturation [19] and maternally provided PGC7 persists into the pre-implantation embryo where PGC7 localises to the pronuclei [20]. Zygotes derived from PGC7 KO oocytes failed to develop into blastocysts, indicating that $\mathrm{PGC7}$ is critical during the post-fertilisation

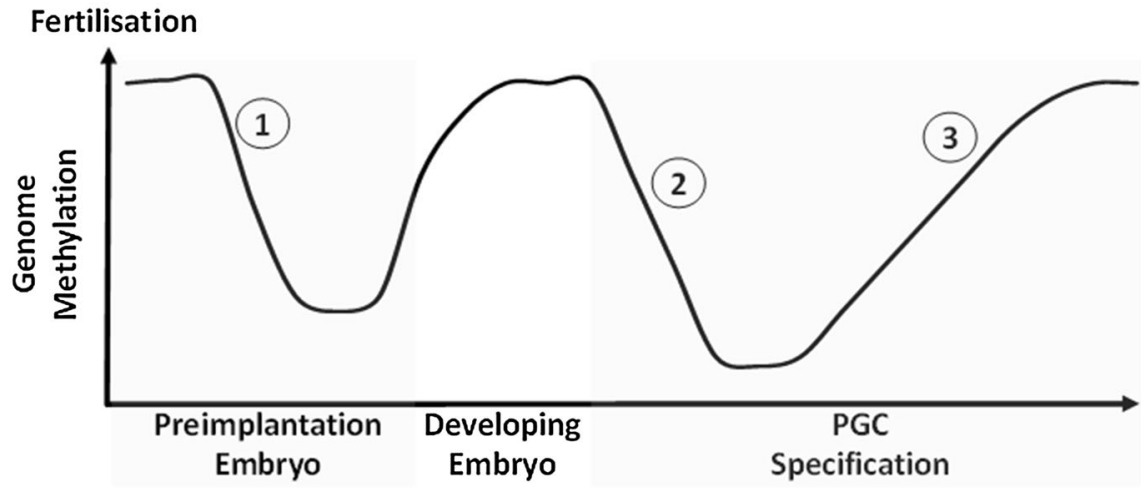

1. Genome-wide demethylation in the preimplantation embryo. Specific protection of imprinted gDMRs.

2. Whole genome demethylation including imprinted gDMRs during PGC specification.

3. Sex-specific methylation. Establishment of methylation at imprinted gDMRs.

Fig. 2 Cycle of methylation through mammalian development. Mammalian cells undergo two major waves of demethylation. The first wave occurs during the pre-implantation period when most methylation, including most sex-specific methylation is cleared, with the exception of the imprinted gDMRs. A second wave of demethylation occurs in PGCs when all methylation is cleared, including gDMRs, except for certain repeats. The genome is then remethylated in a sex specific manner and parental specific methylation at gDMRs is established 
period [20]. This coincides with the timing of genome-wide demethylation and, combined with the genome-specific localisation of PGC7, suggested a role for PGC7 in controlling DNA methylation. In agreement with this, zygotic genomes derived from PGC7 KO oocytes had reduced levels of DNA methylation, with a particularly pronounced effect at the maternally-derived genome [20]. Loss of methylation was observed at a number of maternally methylated DMRs (Peg1, Peg3 and Peg10) as well as two paternally methylated DMRs (Rasgrf1 and H19) and IAP repeats [20].

The specificity of this altered methylation was found to be conferred by the preferential localisation of PGC7 to H3K9me2 modified chromatin [21]. The two parental genomes are not equivalent at fertilisation; the maternal genome is fully chromatinised and enriched for $\mathrm{H} 3 \mathrm{~K} 9 \mathrm{me} 2$ while the paternal genome is subjected to extensive remodelling [22]. The bulk of the genome in mature sperm is packaged around protamines and histones are retained at only a few exceptional regions (1\% in mouse, $10 \%$ in human remain associated with histones) $[23,24]$ including the imprinted DMRs [21]. During the post-fertilisation period, protamines are replaced with histones and the paternal-derived genome is subjected to active demethylation [14] via Tet3-mediated conversion of 5-methylcytosine $(5 \mathrm{mC})$ to 5-hydroxymethylcytosine $(5 \mathrm{hmC})$ [25-27]. The paternally methylated DMRs and the maternal-derived genome are largely protected from active demethylation and PGC7 plays an important role in this process [21].

The preferential binding of PGC7 to the maternalderived genome suggested that the $\mathrm{H} 3 \mathrm{~K} 9$ me2 modification may be important for PGC7 localisation. Consistent with this, PGC7 was found to interact most strongly with H3K9me2 modified histone peptides in in vitro binding assays and a pull-down of endogenous PGC7 demonstrated an enrichment for H3K9me2 [21]. Furthermore, both knockdown of G9a (a lysine methyltransferase which catalyses H3K9me2) and forced expression of Jhmd2a (an H3K9me2 specific demethylase) was found to abrogate the preferential localisation of PGC7 to the maternal genome [21]. Lastly, the two paternal DMRs (Rasgrf1 and H19) which were hypomethylated in $\mathrm{PGC7} \mathrm{KO}$ embryos were enriched for $\mathrm{H} 3 \mathrm{~K} 9 \mathrm{me} 2$ in mature sperm [21], suggesting that $\mathrm{H} 3 \mathrm{~K} 9 \mathrm{me} 2$ mediated binding of PGC7 protects these regions against active demethylation during the post-fertilisation period.

Forced expression of Jhmd2a resulted in strong binding of Tet 3 to both maternal and paternal derived genomes and knockout of PGC7 resulted in abnormal Tet3 mediated conversion of $5 \mathrm{mC}$ to $5 \mathrm{hmC}$ on the maternal-derived genome. Furthermore, recombinant PGC7 was found to directly repress the enzymatic activity of Tet 3 in in vitro

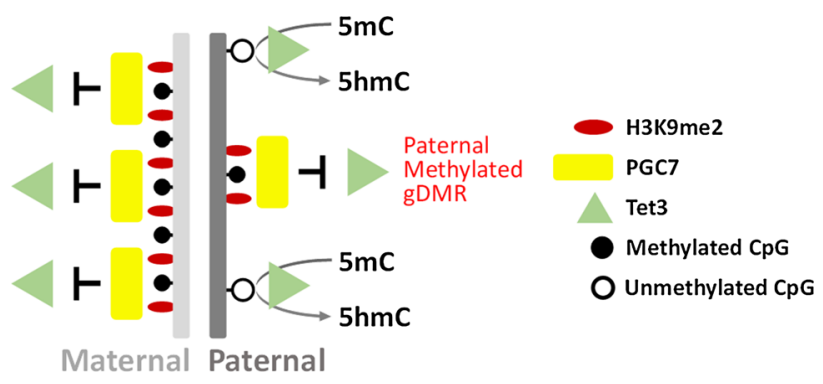

Fig. 3 PCG7 binding is dependent on $\mathrm{H} 3 \mathrm{~K} 9 \mathrm{me} 2$ and protects against Tet3-mediated active demethylation. PGC7 localises to the maternal genome and paternally methylated gDMRs in pre-implantation embryos. Localisation of PGC7 is dependent on $\mathrm{H} 3 \mathrm{~K} 9 \mathrm{me} 2$ which coats the maternal genome but is largely absent on the paternal genome with the exception of methylated gDMRs. Binding of PGC7 protects the maternal genome and paternally methylated gDMRs from Tet3-mediated demethylation

assays [21]. Combined, these results suggest that the H3K9me2 modification attracts PGC7 to inhibit Tet3 demethylation during early embryonic development. This appears to be a general process which is important for preserving the integrity of the maternal genome but is also important for maintaining the DNA methylation of a number of maternal and paternal DMRs (Fig. 3).

\section{Zfp57 recruits the KAP1 co-repressor to methylated DMRs}

In addition to PGC7 a second protein, Zfp57, is known to play an important role in maintaining DNA methylation at imprinted DMRs in early embryos. Zfp57 is one of hundreds of KRAB-ZFPs which are present in mammalian genomes [28]. Each KRAB-ZFP is comprised of a zinc finger domain which binds DNA, often in a sequence specific manner, and a Kruppel Associated Box (KRAB) domain which recruits the KAP1 (aka Trim28) co-repressor complex [28]. KAP1 is a scaffold protein which interacts with a number of heterochromatin proteins including DNMTs [29], Setdb1 (aka ESET) and Heterochromatin Protein 1 (HP1) [30]. The KRAB-ZFP mediated targeting of KAP1 to specific genomic regions, is therefore able to induce heterochromatin formation and transcriptional silencing. At imprinted DMRs, the KAP1 co-repressor complex is recruited by $\mathrm{Zfp} 57$ and this interaction is important for maintaining methylation through the preimplantation period. The role of Zfp57 in imprinting came to light when mutations in Zfp57 were identified in cases of transient neonatal diabetes; this was associated with hypomethylation of multiple imprinted loci [31]. Zfp57 is highly expressed in ES cells and downregulated upon differentiation [32]. In adult tissues, expression of Zfp57 is primarily restricted to testes and ovaries, reflecting its 
critical role in embryogenesis [33]. Maternal-zygotic loss of Zfp57 resulted in no live births, while zygotic KO of Zfp57 resulted in partial lethality [33]. This demonstrated that maternally provided Zfp57 was able to partly compensate for loss of zygotic Zfp57 and indicates that Zfp57 is critical during early embryogenesis.

ChIP-seq revealed that Zfp57 was bound to all known imprinted gDMRs and this binding was specific to the methylated allele [34]. In silico analyses identified a TGCCGC/(N) [29, 34] motif under Zfp57 binding sites and this was confirmed in gel-shift assays performed with the zinc finger domain of Zfp57 [29]. This fragment of Zfp57 was found to bind specifically to the TGCCGC sequence with a particular preference for the methylated motif [29]. The heptamer motif is found at all mouse gDMRs as well as some human gDMRs [29] and may contribute to the selective targeting of these regions for protection against demethylation. Knockout of Zfp57 in embryos or ES cells resulted in loss of methylation at a number of imprinted gDMRs [29, 33, 35] and re-expression of exogenous Zfp57 in null ES cells was unable to restore methylation [35]. This suggests that the continued presence of Zfp57 is required for maintaining methylation at gDMRs in mouse ES cells.

This process is dependent on Zfp57 recruitment of KAP1 to methylated gDMRs as expressing a KAP1-interaction defective, KRAB-domain deleted Zfp57, is unable to rescue the knockout [35]. KAP1 is a scaffold protein which is able to induce de novo DNA methylation through the recruitment of multiple heterochromatin proteins. KAP1 interacts with both de novo (DNMT3a/b) and maintenance (DNMT1) methyltransferases [29] and targeting KAP1 to specific genomic sites is sufficient to induce DNA methylation [36]. In addition, KAP1 is also able to recruit heterochromatin protein 1 (HP1) and Setdb1, a lysine methyltransferase which catalyses $\mathrm{H} 3 \mathrm{~K} 9 \mathrm{me} 3$ [30]. Given the strong association between $\mathrm{H} 3 \mathrm{~K} 9 \mathrm{me} 3$ and DNA methylation, it is likely that the KAP1/Setdb1/H3K9me3 axis is also important for the maintenance of DNA methylation at these regions. The Zfp57 mediated recruitment of the KAP1 co-repressor complex to methylated

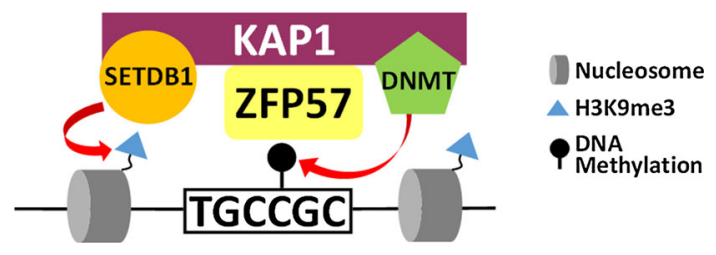

Fig. 4 Zfp57 recruits the KAP1 co-repressor complex to maintain silent modifications. Zfp57 binds preferentially to a methylated motif (TGCCGC/N) found at imprinted gDMRs. Zfp57 recruits the KAP1 scaffold protein which interacts with both DNMT and Setdb1, which catalyse DNA methylation and $\mathrm{H} 3 \mathrm{~K} 9 \mathrm{me} 3$ respectively
gDMRs is therefore able to attract heterochromatin proteins to maintain DNA methylation at these sites (Fig. 4).

\section{Chromatin modifications at imprinted DMRs}

DNA methylation does not occur in isolation, but is instead inextricably linked to histone modifications. Three well characterised histone modifications- $\mathrm{H} 3 \mathrm{~K} 4 \mathrm{me} 3$, H3K9me3 and H3K36me3-are particularly important in the context of genomic imprinting. H3K4me3 is a modification which is highly enriched around gene promoters and is permissive to transcription [37]. At imprinted DMRs, H3K4me3 is restricted to the unmethylated allele $[8,9]$ and the absence of $\mathrm{H} 3 \mathrm{~K} 4 \mathrm{me} 3$ is a specific requirement for the establishment of DNA methylation at a number of maternally methylated gDMRs. Knockout of a H3K4-specific demethylase, LSD2, led to accumulation of $\mathrm{H} 3 \mathrm{~K} 4 \mathrm{me} 2 / 3$ in oocytes and prevented the methylation of several maternal gDMRs [38]. This phenomenon can be explained by the ATRX-DNMT3-DNMT3L (ADD) domain found in DNMT3a/b and the DNMT3L co-factor, which guides the localisation of these methyltransferases. This ADD domain of DNMT3L has been demonstrated to bind to the histone $\mathrm{H} 3$ tail only when the $\mathrm{H} 3 \mathrm{~K} 4$ residue is unmethylated [39]. In DNMT3a/b, the ADD domain also interacts with the $\mathrm{H} 3$ tail and the catalytic activity of these methyltransferases is inhibited by $\mathrm{H} 3 \mathrm{~K} 4 \mathrm{me} 3$ [40]. The localisation and activity of DNMT3a/b, and DNA methylation, is therefore dependent on the absence of $\mathrm{H} 3 \mathrm{~K} 4 \mathrm{me} 3$.

In addition to the specific requirement for $\mathrm{H} 3 \mathrm{~K} 4 \mathrm{me} 0$, the localisation of DNMT3a/b are also guided by interactions with HP1 [41, 42] which is in turn, dependent on H3K9me3 $[43,44]$. The H3K9me3 modification is generally associated with heterochromatin and is particularly prominent at repetitive DNA such as the centromeric, pericentric and telomeric regions [45-47]. This modification is predominantly catalysed by the Suv39h methyltransferases at repeats $[42,47]$ and by Setdb 1 at genic regions $[48,49]$, including the methylated DMRs [50]. The presence of $\mathrm{H} 3 \mathrm{~K} 9 \mathrm{me} 3$ provides a binding site for HP1 $[43,44]$ which then recruits DNMT3a/ b to catalyse DNA methylation [41, 42]. The H3K9me3 modification is therefore able to prime genomic regions for DNA methylation. The Zfp57 mediated recruitment of KAP1 to methylated gDMRs facilitates the maintenance of both $\mathrm{H} 3 \mathrm{~K} 9 \mathrm{me} 3$ via Setdb1 and DNA methylation via interactions with DNMT3a/b, thereby ensuring the retention of DNA methylation at these sites.

In addition to the ADD domain, DNMT3a/b also harbours a PWWP domain which is able to interact with the H3K36me3 modification [51-53]. H3K36me3 is found within the bodies of active genes and is thought to prevent the aberrant initiation of transcription [54, 55]. The 
maternally methylated gDMRs are all $\mathrm{CpG}$ islands which are located within the body of a transcriptional unit and as such, are all enriched for H3K36me3 [9]. In addition to recruiting DNMTs, H3K36me3 also recruits the KDM5B H3K4me3-demethylase which protects these intragenic CGIs from aberrant histone modifications thereby facilitating the continued maintenance of silencing at these sites [56]. The double enrichment of H3K9me3 and H3K36me3 guide the binding of DNMT3a/b via the ADD and PWWD domains respectively, while $\mathrm{H} 3 \mathrm{~K} 36 \mathrm{me} 3$ recruitment of KDM5B protects against the gain of $\mathrm{H} 3 \mathrm{~K} 4 \mathrm{me} 3$ at the intragenic CGIs which comprise maternally methylated DMRs.

\section{Role of transcription and non-coding RNAs in imprinted gene expression}

The association between transcription and DNA methylation at imprinted gDMRs is further supported by studies on the corresponding non-imprinted allele. Many imprinted gene clusters express at least one long non-coding RNA (lncRNA) which is often arranged in the antisense orientation with respect to the methylated gDMR promoter (Fig. 5). Examples of this include the Igf2r/Airn [57], Gnas/Nespas [58], Snrpn/Snrpnlt [59], and the Kcnq1/Kcnq1ot1 loci [60]. At these regions, DNA methylation and silencing modifications suppresses the expression of the ncRNA from the maternal allele. However, the unmethylated paternal allele transcribes a lncRNA which has been associated with methylation and silencing of flanking genes in the imprinted cluster [61]. The exact mechanisms which drive this process are not well understood due to difficulties in separating the function of the ncRNA transcript from the

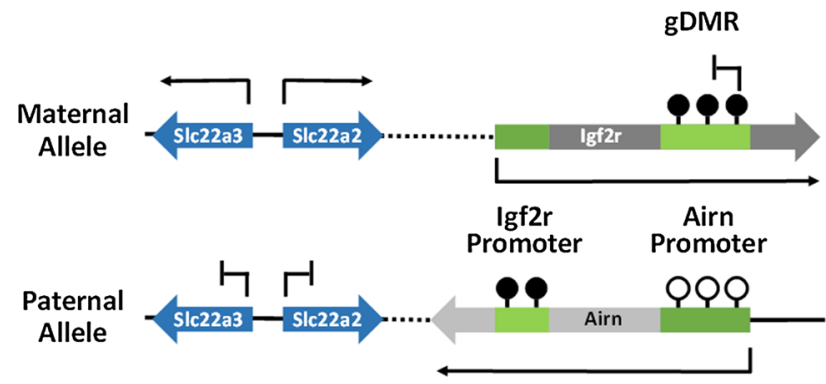

Fig. 5 Example of non-coding RNA and transcription in imprinted gene expression. The Igf2r/Airn imprinted locus is an example locus which illustrates the role of transcription and non-coding RNA in controlling expression of imprinted genes. The maternal methylation at the gDMR prevents expression of the non-coding Airn transcript from the maternal allele. The gDMR is unmethylated at the paternal allele which allows expression of the Airn transcript. Transcription of Airn through the Igf2r promoter results in methylation at the paternal allele. The non-coding Airn transcript prevents expression of Slc22a2 and Slc22a3 from the paternal allele process of transcription. Nonetheless, experiments where the ncRNA transcript was artificially truncated with the addition of a premature Poly-A signal or destabilising the transcript, have demonstrated that the ncRNA transcript is involved in specifying imprinted gene expression at some clusters $[62,63]$.

In addition, several other studies have indicated that the process of transcription may also play a role in this process $[64,65]$. For example, at the Igf2r locus, transcription of the Airn ncRNA was demonstrated to be important for methylation and silencing of the Igf $2 \mathrm{r}$ promoter on the paternal allele, independent of the Airn lncRNA [64]. In this study, a truncated Airn transcript was inserted in the reverse orientation so that transcription no longer traversed the Igf $2 r$ promoter. This arrangement preserved the expression of a truncated Airn transcript while abolishing transcription across the Igf $2 \mathrm{r}$ promoter. The Igf2r promoter was normally methylated in a control line where the truncated Airn promoter was positioned in the forward orientation but methylation was lost when the Airn promoter was positioned in the reverse orientation [64]. This effectively demonstrates that both the IncRNA products and the process of transcription across promoters contributes to imprinted gene expression.

\section{ATRX/Daxx/H3.3 maintain H3K9me3 at methylated DMRs}

The active transcription through the intragenic maternal methylated DMRs poses an additional challenge for the maintenance of methylation at these sites. The position of these imprinted DMRs means that these heterochromatic foci are subjected to transcription, and chromatin modifications must be continuously restored following the passage of RNA polymerase. This process has recently been demonstrated to be dependent on a histone variant, H3.3. Unlike the canonical histones (H3.1/H3.2), which are expressed and deposited only during S-phase, histone H3.3 is expressed throughout the cell cycle and replaces histones which are displaced by transcription [66, 67]. Consistent with this, ChIP-seq revealed that $\mathrm{H} 3.3$ predominantly localises to the promoters and gene bodies of active genes [68]. However, in mouse ES cells, H3.3 enrichment was also detected at a number of heterochromatic regions including telomeres, ERVs and imprinted DMRs [12, 68, 69]. Allele-specific ChIP-seq and ChIP-PCR revealed that H3.3 was preferentially enriched on the DNA methylated allele at all imprinted DMRs tested, suggesting a functional role for H3.3 in maintaining silencing at the methylated allele [12].

Two major chaperone complexes which deposit H3.3 have been identified. The HIRA complex is the major H3.3 chaperone at euchromatin genic sites $[68,70]$ while 


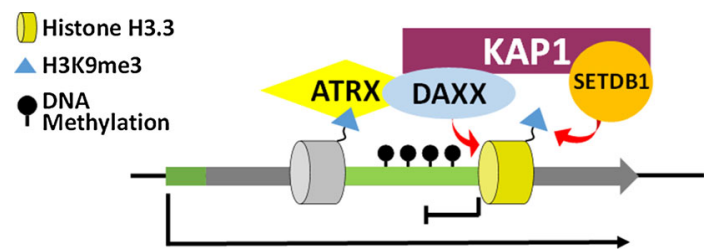

Fig. 6 The ATRX/Daxx/H3.3 complex continuously maintain heterochromatin at imprinted DMRs. ATRX can directly bind H3K9me3 at imprinted DMRs to recruit Daxx and deposit the replicationindependent H3.3 histone variant. Daxx interacts with the KAP1 corepressor complex which recruits Setdb1 to catalyse H3K9me3 heterochromatin at these sites. The replication-independent nature of H3.3 ensures the continuous maintenance of heterochromatin at gDMRs throughout the cell cycle particularly during transcription

deposition of $\mathrm{H} 3.3$ at heterochromatin is dependent on the ATRX/Daxx complex [68, 71]. Daxx is the H3.3 chaperone in this complex [72-74] while ATRX is a chromatin remodeller, and both components are required for the incorporation of $\mathrm{H} 3.3$ at heterochromatin, including methylated DMRs. ChIP-seq revealed ATRX enrichment at a number of imprinted DMRs in mouse ES cells and allele-specific ChIP-PCR demonstrated preferential localisation to the methylated allele [12]. Similar to DNMT3, ATRX also harbours an ADD domain which specifically recognises $\mathrm{H} 3 \mathrm{~K} 4 \mathrm{me} 0$ [75-77] but additionally it directly binds H3K9me3 [75-77]. ATRX also contains an HP1 binding motif (67). These interactions likely promote the specific localisation of ATRX/Daxx/H3.3 to the methylated DMR to facilitate allele-specific deposition of H3.3. Knockout of ATRX led to loss of H3.3 at imprinted DMRs which was co-incident with the loss of the H3K9me3 heterochromatin modification [12]. A similar phenomenon was observed at telomeres [78] and ERV repeats [69] where ChIP-reChIP assays demonstrated that H3.3 is modified with K9me3 (H3.3K9me3) [69, 78]. Furthermore, Daxx was shown to interact with KAP1 and Setdb1 was required for catalysing $\mathrm{H} 3.3 \mathrm{~K} 9 \mathrm{me} 3$ at ERVs [69]. It is likely that an equivalent pathway operates at methylated DMRs where ATRX/Daxx deposits H3.3 which is modified to $\mathrm{H} 3.3 \mathrm{~K} 9 \mathrm{me} 3$ by Setdb1. In this case, KAP1/Setdb1 could be recruited either by Zfp57 or Daxx, or a combination of both. The replication-independent nature of H3.3 would ensure continued maintenance of heterochromatin at methylated DMRs throughout the cell cycle and particularly when challenged by transcription (Fig. 6).

\section{Maintenance pathways are active at non-imprinted genomic regions}

In addition to the imprinted DMRs, a number of other methylated genomic sites are also targeted by Zfp57 and the ATRX/Daxx complex [12, 34, 69]. This includes the
IAP retrotransposons which are heavily methylated and continuously protected from demethylation, even in primordial germ cells $[15,16]$. In addition, both Zfp57 and ATRX/Daxx were found to localise with other heterochromatin modified regions including the $3^{\prime}$ end of zinc finger genes [12, 34]. Both IAP retrotransposons and the GC-rich $3^{\prime}$ intragenic zinc finger genes are also silenced by the KAP1 co-repressor complex [79, 80]. At IAP elements, KAP1 recruitment is predominantly dependent on Zfp809 [79]; however, Zfp57 and the ATRX/Daxx complex may provide an additional mode of recruitment or further stabilise the KAP1 interaction at IAPs. Given that aberrant expression of IAPs is deleterious to the genome [81], multiple redundant mechanisms for silencing would be important for ensuring genome integrity.

Similar to IAPs and imprinted methylated DMRs, the $3^{\prime}$ end of zinc finger genes are also enriched for heterochromatin modifications [82, 83]. This intragenic GC-rich regions may have the capacity to act as promoters and heterochromatin modifications would be required to prevent aberrant transcriptional initiation from these intragenic sites, not unlike the imprinted DMRs. As such, a highly similar complement of chromatin modifications and heterochromatin proteins are observed at these two classes of genes. This includes a combination of H3K36me3, H3K9me3 and DNA methylation which is linked to transcription and the recruitment of KAP1 respectively [8, 9, $80,82]$. In addition, the $3^{\prime}$ end of zinc finger genes are also enriched for HP1 and Zfp57 which presumably recruits KAP1 to these sites, as well as the ATRX/Daxx/H3.3 complex which can reinforce heterochromatin modifications throughout the cell cycle [12, 34]. The pathways for maintaining genomic imprinting are therefore also required for preserving heterochromatin at other non-imprinted genomic regions which nonetheless share features with the imprinted DMRs.

\section{Summary}

The phenomenon of genomic imprinting is therefore dependent on the acquisition of differential methylation in parental germlines followed by the selective maintenance of imprints through development. Sex-specific differences during germline specification results in a differential pattern of DNA methylation in parental genomes at fertilisation. These differentially methylated regions include the imprinted gDMRs but are not exclusive to these sites. The differential methylation at non-imprinted regions is subsequently erased during the pre-implantation period when active and passive demethylation processes occur indiscriminately across the genome, except at regions which are specifically protected. 
The maternal genome and paternally methylated gDMRs are protected from active Tet3 mediated demethylation via $\mathrm{H} 3 \mathrm{~K} 9 \mathrm{me} 2$ dependent recruitment of PGC7. Silencing modifications at gDMRs are then further reinforced by the binding of Zfp57 and the KAP1 co-repressor complex which recruits both DNMT3a/b and Setdb1 to catalyse DNA methylation and H3K9me3 respectively. The continuous protection of silencing modifications at gDMRs is further facilitated by the ATRX/ Daxx complex which deposits the replication independent H3.3 histone variant. Daxx reportedly interacts with KAP1 and Setdb1 which modifies $\mathrm{H} 3.3$ to $\mathrm{H} 3.3 \mathrm{~K} 9 \mathrm{me} 3$, thus ensuring the faithful protection of heterochromatin modifications at gDMRs, even when subjected to transcription. In addition to the methylated gDMRs, both Zfp57 and the ATRX/Daxx/H3.3 complex also bind other methylated regions such as IAP retrotransposons and the $3^{\prime}$ end of zinc finger genes. Thus, neither the acquisition of differential methylation nor the maintenance processes are exclusive to imprinted gDMRs, and neither process is deterministic for genomic imprinting. Instead, the imprinted gDMRs arise from the intersection of these two pathways and it is this combinatorial interaction which ultimately distinguishes imprinted gDMRS from the rest of the genome.

Acknowledgments This work was supported by the Medical Research Council [grant number MC_UU_12025/ unit programme MC_UU_12009/3].

Open Access This article is distributed under the terms of the Creative Commons Attribution 4.0 International License (http:// creativecommons.org/licenses/by/4.0/), which permits unrestricted use, distribution, and reproduction in any medium, provided you give appropriate credit to the original author(s) and the source, provide a link to the Creative Commons license, and indicate if changes were made.

\section{References}

1. Jirtle RL (2012) http://www.geneimprint.com/. Accessed 28 Oct 2015

2. Thorvaldsen JL, Duran KL, Bartolomei MS (1998) Deletion of the H19 differentially methylated domain results in loss of imprinted expression of H19 and Igf2. Genes Dev 12:3693-3702

3. Yang T, Adamson TE, Resnick JL, Leff S, Wevrick R, Francke U, Jenkins NA, Copeland NG, Brannan CI (1998) A mouse model for Prader-Willi syndrome imprinting-centre mutations. Nat Genet 19:25-31

4. Fitzpatrick GV, Soloway PD, Higgins MJ (2002) Regional loss of imprinting and growth deficiency in mice with a targeted deletion of KvDMR1. Nat Genet 32:426-431

5. Lin SP, Youngson N, Takada S, Seitz H, Reik W, Paulsen M, Cavaille J, Ferguson-Smith AC (2003) Asymmetric regulation of imprinting on the maternal and paternal chromosomes at the Dlk1-Gt12 imprinted cluster on mouse chromosome 12. Nat Genet 35:97-102
6. Kelsey G, Feil R (2013) New insights into establishment and maintenance of DNA methylation imprints in mammals. Philos Trans R Soc Lond B Biol Sci 368:20110336

7. Regha K, Sloane MA, Huang R, Pauler FM, Warczok KE, Melikant B, Radolf M, Martens JH, Schotta G, Jenuwein T, Barlow DP (2007) Active and repressive chromatin are interspersed without spreading in an imprinted gene cluster in the mammalian genome. Mol Cell 27:353-366

8. Singh P, Wu X, Lee DH, Li AX, Rauch TA, Pfeifer GP, Mann JR, Szabo PE (2011) Chromosome-wide analysis of parental allele-specific chromatin and DNA methylation. Mol Cell Biol 31:1757-1770

9. Mikkelsen TS, Ku M, Jaffe DB, Issac B, Lieberman E, Giannoukos G, Alvarez P, Brockman W, Kim TK, Koche RP, Lee W, Mendenhall E, O'donovan A, Presser A, Russ C, Xie X, Meissner A, Wernig M, Jaenisch R, Nusbaum C, Lander ES, Bernstein BE (2007) Genome-wide maps of chromatin state in pluripotent and lineage-committed cells. Nature 448:553-560

10. Fournier C, Goto Y, Ballestar E, Delaval K, Hever AM, Esteller M, Feil R (2002) Allele-specific histone lysine methylation marks regulatory regions at imprinted mouse genes. EMBO J 21:6560-6570

11. Andergassen D, Dotter CP, Kulinski TM, Guenzl PM, Bammer PC, Barlow DP, Pauler FM, Hudson QJ (2015) Allelome.PRO, a pipeline to define allele-specific genomic features from highthroughput sequencing data. Nucleic Acids Res 43:e146

12. Voon HP, Hughes JR, Rode C, De La Rosa-Velazquez IA, Jenuwein T, Feil R, Higgs DR, Gibbons RJ (2015) ATRX plays a key role in maintaining silencing at interstitial heterochromatic loci and imprinted genes. Cell Rep 11:405-418

13. Sasaki H, Matsui Y (2008) Epigenetic events in mammalian germ-cell development: reprogramming and beyond. Nat Rev Genet 9:129-140

14. Mayer W, Niveleau A, Walter J, Fundele R, Haaf T (2000) Demethylation of the zygotic paternal genome. Nature 403:501-502

15. Hajkova P, Erhardt S, Lane N, Haaf T, El-Maarri O, Reik W, Walter J, Surani MA (2002) Epigenetic reprogramming in mouse primordial germ cells. Mech Dev 117:15-23

16. Guibert S, Forne T, Weber M (2012) Global profiling of DNA methylation erasure in mouse primordial germ cells. Genome Res 22:633-641

17. Smith ZD, Chan MM, Mikkelsen TS, Gu H, Gnirke A, Regev A, Meissner A (2012) A unique regulatory phase of DNA methylation in the early mammalian embryo. Nature 484:339-344

18. Smallwood SA, Tomizawa S, Krueger F, Ruf N, Carli N, Segonds-Pichon A, Sato S, Hata K, Andrews SR, Kelsey G (2011) Dynamic CpG island methylation landscape in oocytes and preimplantation embryos. Nat Genet 43:811-814

19. Sato M, Kimura T, Kurokawa K, Fujita Y, Abe K, Masuhara M, Yasunaga T, Ryo A, Yamamoto M, Nakano T (2002) Identification of PGC7, a new gene expressed specifically in preimplantation embryos and germ cells. Mech Dev 113:91-94

20. Nakamura $T$, Arai $Y$, Umehara $H$, Masuhara M, Kimura $T$, Taniguchi H, Sekimoto T, Ikawa M, Yoneda Y, Okabe M, Tanaka S, Shiota K, Nakano T (2007) PGC7/Stella protects against DNA demethylation in early embryogenesis. Nat Cell Biol 9:64-71

21. Nakamura T, Liu YJ, Nakashima $H$, Umehara $H$, Inoue $K$, Matoba S, Tachibana M, Ogura A, Shinkai Y, Nakano T (2012) PGC7 binds histone H3K9me2 to protect against conversion of $5 \mathrm{mC}$ to $5 \mathrm{hmC}$ in early embryos. Nature $486: 415-419$

22. Santos F, Peters AH, Otte AP, Reik W, Dean W (2005) Dynamic chromatin modifications characterise the first cell cycle in mouse embryos. Dev Biol 280:225-236 
23. Hammoud SS, Nix DA, Zhang H, Purwar J, Carrell DT, Cairns BR (2009) Distinctive chromatin in human sperm packages genes for embryo development. Nature 460:473-478

24. Brykczynska U, Hisano M, Erkek S, Ramos L, Oakeley EJ, Roloff TC, Beisel C, Schubeler D, Stadler MB, Peters AH (2010) Repressive and active histone methylation mark distinct promoters in human and mouse spermatozoa. Nat Struct Mol Biol 17:679-687

25. Iqbal K, Jin SG, Pfeifer GP, Szabo PE (2011) Reprogramming of the paternal genome upon fertilization involves genome-wide oxidation of 5-methylcytosine. Proc Natl Acad Sci USA 108:3642-3647

26. Peat JR, Dean W, Clark SJ, Krueger F, Smallwood SA, Ficz G, Kim JK, Marioni JC, Hore TA, Reik W (2014) Genome-wide bisulfite sequencing in zygotes identifies demethylation targets and maps the contribution of TET3 oxidation. Cell Rep 9:1990-2000

27. Gu TP, Guo F, Yang H, Wu HP, Xu GF, Liu W, Xie ZG, Shi L, He X, Jin SG, Iqbal K, Shi YG, Deng Z, Szabo PE, Pfeifer GP, Li J, Xu GL (2011) The role of Tet3 DNA dioxygenase in epigenetic reprogramming by oocytes. Nature 477:606-610

28. Wolf G, Greenberg D, Macfarlan TS (2015) Spotting the enemy within: targeted silencing of foreign DNA in mammalian genomes by the Kruppel-associated box zinc finger protein family. Mob DNA 6:17

29. Quenneville S, Verde G, Corsinotti A, Kapopoulou A, Jakobsson J, Offner S, Baglivo I, Pedone PV, Grimaldi G, Riccio A, Trono D (2011) In embryonic stem cells, ZFP57/KAP1 recognize a methylated hexanucleotide to affect chromatin and DNA methylation of imprinting control regions. Mol Cell 44:361-372

30. Schultz DC, Ayyanathan K, Negorev D, Maul GG, Rauscher FJ 3rd (2002) SETDB1: a novel KAP-1-associated histone H3, lysine 9-specific methyltransferase that contributes to HP1-mediated silencing of euchromatic genes by KRAB zinc-finger proteins. Genes Dev 16:919-932

31. MacKay DJ, Callaway JL, Marks SM, White HE, Acerini CL, Boonen SE, Dayanikli P, Firth HV, Goodship JA, Haemers AP, Hahnemann JM, Kordonouri O, Masoud AF, Oestergaard E, Storr J, Ellard S, Hattersley AT, Robinson DO, Temple IK (2008) Hypomethylation of multiple imprinted loci in individuals with transient neonatal diabetes is associated with mutations in ZFP57. Nat Genet 40:949-51

32. Li X, Leder P (2007) Identifying genes preferentially expressed in undifferentiated embryonic stem cells. BMC Cell Biol 8:37

33. Li X, Ito M, Zhou F, Youngson N, Zuo X, Leder P, FergusonSmith AC (2008) A maternal-zygotic effect gene, Zfp57, maintains both maternal and paternal imprints. Dev Cell 15:547-557

34. Strogantsev R, Krueger F, Yamazawa K, Shi H, Gould P, Goldman-Roberts M, Mcewen K, Sun B, Pedersen R, FergusonSmith AC (2015) Allele-specific binding of ZFP57 in the epigenetic regulation of imprinted and non-imprinted monoallelic expression. Genome Biol 16:112

35. Zuo X, Sheng J, Lau HT, Mcdonald CM, Andrade M, Cullen DE, Bell FT, Iacovino M, Kyba M, Xu G, Li X (2012) Zinc finger protein ZFP57 requires its co-factor to recruit DNA methyltransferases and maintains DNA methylation imprint in embryonic stem cells via its transcriptional repression domain. J Biol Chem 287:2107-2118

36. Quenneville S, Turelli P, Bojkowska K, Raclot C, Offner S, Kapopoulou A, Trono D (2012) The KRAB-ZFP/KAP1 system contributes to the early embryonic establishment of site-specific DNA methylation patterns maintained during development. Cell Rep 2:766-773

37. Schneider R, Bannister AJ, Myers FA, Thorne AW, CraneRobinson C, Kouzarides T (2004) Histone H3 lysine 4 methylation patterns in higher eukaryotic genes. Nat Cell Biol 6:73-77
38. Ciccone DN, Su H, Hevi S, Gay F, Lei H, Bajko J, Xu G, Li E, Chen T (2009) KDM1B is a histone H3K4 demethylase required to establish maternal genomic imprints. Nature 461:415-418

39. Ooi SK, Qiu C, Bernstein E, Li K, Jia D, Yang Z, ErdjumentBromage H, Tempst P, Lin SP, Allis CD, Cheng X, Bestor TH (2007) DNMT3L connects unmethylated lysine 4 of histone H3 to de novo methylation of DNA. Nature 448:714-717

40. Li BZ, Huang Z, Cui QY, Song XH, Du L, Jeltsch A, Chen P, Li G, Li E, Xu GL (2011) Histone tails regulate DNA methylation by allosterically activating de novo methyltransferase. Cell Res 21:1172-1181

41. Fuks F, Hurd PJ, Deplus R, Kouzarides T (2003) The DNA methyltransferases associate with HP1 and the SUV39H1 histone methyltransferase. Nucleic Acids Res 31:2305-2312

42. Lehnertz B, Ueda Y, Derijck AA, Braunschweig U, Perez-Burgos L, Kubicek S, Chen T, Li E, Jenuwein T, Peters AH (2003) Suv39h-mediated histone H3 lysine 9 methylation directs DNA methylation to major satellite repeats at pericentric heterochromatin. Curr Biol CB 13:1192-1200

43. Bannister AJ, Zegerman P, Partridge JF, Miska EA, Thomas JO, Allshire RC, Kouzarides T (2001) Selective recognition of methylated lysine 9 on histone H3 by the HP1 chromo domain. Nature 410:120-124

44. Lachner M, O'carroll D, Rea S, Mechtler K, Jenuwein T (2001) Methylation of histone H3 lysine 9 creates a binding site for HP1 proteins. Nature 410:116-120

45. Peters AH, O'carroll D, Scherthan H, Mechtler K, Sauer S, Schofer C, Weipoltshammer K, Pagani M, Lachner M, Kohlmaier A, Opravil S, Doyle M, Sibilia M, Jenuwein T (2001) Loss of the Suv39h histone methyltransferases impairs mammalian heterochromatin and genome stability. Cell 107:323-337

46. Peters AH, Kubicek S, Mechtler K, O'sullivan RJ, Derijck AA, Perez-Burgos L, Kohlmaier A, Opravil S, Tachibana M, Shinkai Y, Martens JH, Jenuwein T (2003) Partitioning and plasticity of repressive histone methylation states in mammalian chromatin. Mol Cell 12:1577-1589

47. Garcia-Cao M, O'sullivan R, Peters AH, Jenuwein T, Blasco MA (2004) Epigenetic regulation of telomere length in mammalian cells by the Suv39h1 and Suv39h2 histone methyltransferases. Nature Genet 36:94-99

48. Dodge JE, Kang YK, Beppu H, Lei H, Li E (2004) Histone H3K9 methyltransferase ESET is essential for early development. Mol Cell Biol 24:2478-2486

49. Bilodeau S, Kagey MH, Frampton GM, Rahl PB, Young RA (2009) SetDB1 contributes to repression of genes encoding developmental regulators and maintenance of ES cell state. Genes Dev 23:2484-2489

50. Leung D, Du T, Wagner U, Xie W, Lee AY, Goyal P, Li Y, Szulwach KE, Jin P, Lorincz MC, Ren B (2014) Regulation of DNA methylation turnover at LTR retrotransposons and imprinted loci by the histone methyltransferase Setdb1. Proc Natl Acad Sci USA 111:6690-6695

51. Dhayalan A, Rajavelu A, Rathert P, Tamas R, Jurkowska RZ, Ragozin S, Jeltsch A (2010) The Dnmt3a PWWP domain reads histone 3 lysine 36 trimethylation and guides DNA methylation. J Biol Chem 285:26114-26120

52. Baubec T, Colombo DF, Wirbelauer C, Schmidt J, Burger L, Krebs AR, Akalin A, Schubeler D (2015) Genomic profiling of DNA methyltransferases reveals a role for DNMT3B in genic methylation. Nature 520:243-247

53. Morselli M, Pastor WA, Montanini B, Nee K, Ferrari R, Fu K, Bonora G, Rubbi L, Clark AT, Ottonello S, Jacobsen SE, Pellegrini M (2015) In vivo targeting of de novo DNA methylation by histone modifications in yeast and mouse. eLife 4:e06205

54. Carrozza MJ, Li B, Florens L, Suganuma T, Swanson SK, Lee KK, Shia WJ, Anderson S, Yates J, Washburn MP, Workman JL 
(2005) Histone H3 methylation by Set2 directs deacetylation of coding regions by Rpd3S to suppress spurious intragenic transcription. Cell 123:581-592

55. Venkatesh S, Smolle M, Li H, Gogol MM, Saint M, Kumar S, Natarajan K, Workman JL (2012) Set2 methylation of histone H3 lysine 36 suppresses histone exchange on transcribed genes. Nature 489:452-455

56. Xie L, Pelz C, Wang W, Bashar A, Varlamova O, Shadle S, Impey S (2011) KDM5B regulates embryonic stem cell self-renewal and represses cryptic intragenic transcription. EMBO J 30:1473-1484

57. Wutz A, Smrzka OW, Schweifer N, Schellander K, Wagner EF, Barlow DP (1997) Imprinted expression of the Igf2r gene depends on an intronic CpG island. Nature 389:745-749

58. Wroe SF, Kelsey G, Skinner JA, Bodle D, Ball ST, Beechey CV, Peters J, Williamson CM (2000) An imprinted transcript, antisense to Nesp, adds complexity to the cluster of imprinted genes at the mouse Gnas locus. Proc Natl Acad Sci USA 97:3342-3346

59. Landers M, Bancescu DL, Le Meur E, Rougeulle C, Glatt-Deeley H, Brannan C, Muscatelli F, Lalande M (2004) Regulation of the large (approximately $1000 \mathrm{~kb}$ ) imprinted murine Ube3a antisense transcript by alternative exons upstream of Snurf/Snrpn. Nucleic Acids Res 32:3480-3492

60. Smilinich NJ, Day CD, Fitzpatrick GV, Caldwell GM, Lossie AC, Cooper PR, Smallwood AC, Joyce JA, Schofield PN, Reik W, Nicholls RD, Weksberg R, Driscoll DJ, Maher ER, Shows TB, Higgins MJ (1999) A maternally methylated CpG island in KvLQT1 is associated with an antisense paternal transcript and loss of imprinting in Beckwith-Wiedemann syndrome. Proc Natl Acad Sci USA 96:8064-8069

61. Koerner MV, Pauler FM, Huang R, Barlow DP (2009) The function of non-coding RNAs in genomic imprinting. Development 136:1771-1783

62. Nagano T, Mitchell JA, Sanz LA, Pauler FM, Ferguson-Smith AC, Feil R, Fraser P (2008) The Air noncoding RNA epigenetically silences transcription by targeting G9a to chromatin. Science 322:1717-1720

63. Pandey RR, Mondal T, Mohammad F, Enroth S, Redrup L, Komorowski J, Nagano T, Mancini-Dinardo D, Kanduri C (2008) Kcnq1ot1 antisense noncoding RNA mediates lineage-specific transcriptional silencing through chromatin-level regulation. Mol Cell 32:232-246

64. Latos PA, Pauler FM, Koerner MV, Senergin HB, Hudson QJ, Stocsits RR, Allhoff W, Stricker SH, Klement RM, Warczok KE, Aumayr K, Pasierbek P, Barlow DP (2012) Airn transcriptional overlap, but not its lncRNA products, induces imprinted Igf2r silencing. Science 338:1469-1472

65. Smith EY, Futtner CR, Chamberlain SJ, Johnstone KA, Resnick JL (2011) Transcription is required to establish maternal imprinting at the Prader-Willi syndrome and Angelman syndrome locus. PLoS Genet 7:e1002422

66. Ahmad K, Henikoff S (2002) The histone variant H3.3 marks active chromatin by replication-independent nucleosome assembly. Mol Cell 9:1191-1200

67. Tagami H, Ray-Gallet D, Almouzni G, Nakatani Y (2004) Histone H3.1 and H3.3 complexes mediate nucleosome assembly pathways dependent or independent of DNA synthesis. Cell 116:51-61

68. Goldberg AD, Banaszynski LA, Noh KM, Lewis PW, Elsaesser SJ, Stadler S, Dewell S, Law M, Guo X, Li X, Wen D, Chapgier A, Dekelver RC, Miller JC, Lee YL, Boydston EA, Holmes MC, Gregory PD, Greally JM, Rafii S, Yang C, Scambler PJ, Garrick D, Gibbons RJ, Higgs DR, Cristea IM, Urnov FD, Zheng D, Allis
CD (2010) Distinct factors control histone variant H3.3 localization at specific genomic regions. Cell 140:678-691

69. Elsasser SJ, Noh KM, Diaz N, Allis CD, Banaszynski LA (2015) Histone H3.3 is required for endogenous retroviral element silencing in embryonic stem cells. Nature 522:240-244

70. Ray-Gallet D, Quivy JP, Scamps C, Martini EM, Lipinski M, Almouzni G (2002) HIRA is critical for a nucleosome assembly pathway independent of DNA synthesis. Mol Cell 9:1091-1100

71. Wong LH, Mcghie JD, Sim M, Anderson MA, Ahn S, Hannan RD, George AJ, Morgan KA, Mann JR, Choo KH (2010) ATRX interacts with $\mathrm{H} 3.3$ in maintaining telomere structural integrity in pluripotent embryonic stem cells. Genome Res 20:351-360

72. Denizio JE, Elsasser SJ, Black BE (2014) DAXX co-folds with H3.3/H4 using high local stability conferred by the H3.3 variant recognition residues. Nucleic Acids Res 42:4318-4331

73. Elsasser SJ, Huang H, Lewis PW, Chin JW, Allis CD, Patel DJ (2012) DAXX envelops a histone H3.3-H4 dimer for H3.3specific recognition. Nature 491:560-565

74. Liu CP, Xiong C, Wang M, Yu Z, Yang N, Chen P, Zhang Z, Li G, Xu RM (2012) Structure of the variant histone H3.3-H4 heterodimer in complex with its chaperone DAXX. Nat Struct Mol Biol 19:1287-1292

75. Dhayalan A, Tamas R, Bock I, Tattermusch A, Dimitrova E, Kudithipudi S, Ragozin S, Jeltsch A (2011) The ATRX-ADD domain binds to $\mathrm{H} 3$ tail peptides and reads the combined methylation state of K4 and K9. Hum Mol Genet 20:2195-2203

76. Eustermann S, Yang JC, Law MJ, Amos R, Chapman LM, Jelinska C, Garrick D, Clynes D, Gibbons RJ, Rhodes D, Higgs DR, Neuhaus D (2011) Combinatorial readout of histone H3 modifications specifies localization of ATRX to heterochromatin. Nat Struct Mol Biol 18:777-782

77. Iwase S, Xiang B, Ghosh S, Ren T, Lewis PW, Cochrane JC, Allis CD, Picketts DJ, Patel DJ, Li H, Shi Y (2011) ATRX ADD domain links an atypical histone methylation recognition mechanism to human mental-retardation syndrome. Nat Struct Mol Biol 18:769-776

78. Udugama M, Chang FT, Chan FL, Tang MC, Pickett HA, Mcghie JD, Mayne L, Collas P, Mann JR, Wong LH (2015) Histone variant $\mathrm{H} 3.3$ provides the heterochromatic H3 lysine 9 trimethylation mark at telomeres. Nucleic Acids Res 43:10227-10237

79. Wolf G, Yang P, Fuchtbauer AC, Fuchtbauer EM, Silva AM, Park C, Wu W, Nielsen AL, Pedersen FS, Macfarlan TS (2015) The KRAB zinc finger protein ZFP809 is required to initiate epigenetic silencing of endogenous retroviruses. Genes Dev 29:538-554

80. O'geen H, Squazzo SL, Iyengar S, Blahnik K, Rinn JL, Chang HY, Green R, Farnham PJ (2007) Genome-wide analysis of KAP1 binding suggests autoregulation of KRAB-ZNFs. PLoS Genet 3:e89

81. Crichton JH, Dunican DS, Maclennan M, Meehan RR, Adams IR (2014) Defending the genome from the enemy within: mechanisms of retrotransposon suppression in the mouse germline. Cell Mol Life Sci CMLS 71:1581-1605

82. Blahnik KR, Dou L, Echipare L, Iyengar S, O'geen H, Sanchez E, Zhao Y, Marra MA, Hirst M, Costello JF, Korf I, Farnham PJ (2011) Characterization of the contradictory chromatin signatures at the $3^{\prime}$ exons of zinc finger genes. PloS One 6:e17121

83. Hahn MA, Wu X, Li AX, Hahn T, Pfeifer GP (2011) Relationship between gene body DNA methylation and intragenic H3K9me3 and H3K36me3 chromatin marks. PLoS One 6:e18844 